\title{
Complexity of four-dimensional hairy anti-de-Sitter black holes with a rotating string and shear viscosity in generalized scalar-tensor theories
}

\author{
Moisés Bravo-Gaete ${ }^{1, \mathrm{a}} \mathbb{C}$, F. F. Santos ${ }^{2,3, \mathrm{~b}}$ \\ ${ }^{1}$ Facultad de Ciencias Básicas, Universidad Católica del Maule, Casilla 617, Talca, Chile \\ 2 Departamento de Física, Universidade Federal da Paraíba, Caixa Postal 5008, João Pessoa, Paraíba 58051-970, Brazil \\ ${ }^{3}$ Instituto Federal de Educação, Ciência e Tecnologia do Sertão Pernambucano, Campus Petrolina Zona Rural, Petrolina, Pernambuco 56316-686, \\ Brazil
}

Received: 27 February 2021 / Accepted: 25 January 2022 / Published online: 2 February 2022

(C) The Author(s) 2022

\begin{abstract}
In four dimensions, we consider a generalized scalar-tensor theory where the coupling functions only depend on the kinetic term of the scalar field. For this model, we obtain a set of hairy anti-de-Sitter black hole solutions, allowing us to calculate the computational complexity, according to the Complexity equals Action conjecture. To perform this, the system contains a particle moving on the boundary, corresponding to the insertion of a fundamental string in the bulk. The effect string is given by the Nambu-Goto term, analyzing the time development of this system. Together with the above, we calculate the shear viscosity, where the viscosity/entropy density ratio can violate the Kovtun-Son-Starinets bound for a suitable choice of coupling functions.
\end{abstract}

\section{Introduction}

In recent years, there is a deep interest in the application to black hole physics from computational complexity ${ }^{1}$ (see for example [1-7]), thanks to the seminal ideas from the quantum information process [8] as well as arguments for the existence of a second law of complexity, behaving similar to the entropy [9]. In particular, and based on the works [1012], the growth in complexity is related to problems of black holes, such as information problem, the existence of firewalls, or transparency of horizons.

To connect quantum complexity and black holes, in $[13,14]$ the authors provide the Complexity equals Action

\footnotetext{
a e-mail: mbravo@ucm.cl (corresponding author)

be-mail: fabiano.ffs23@gmail.com

1 Defined as the number of necessary gates which operate to produce the target state from the reference state.
}

(CA) conjecture, where the complexity is dual to the bulk action on a certain region of the spacetime denominated as Wheeler-de Witt patch (WdW). A generalization of this conjecture can be performed for systems other than stationary states, such as a system powered by a drag force [2]. For this work, the idea is to consider a Wilson line operator located on a five-dimensional anti-de-Sitter $\left(\mathrm{AdS}_{5}\right)$ spacetime, inserting a fundamental string, being reflected by the addition of a Nambu-Goto (NG) term to the Einstein-Hilbert action together with a cosmological constant.

This procedure has been explored for the three dimensional case [1], studying the effects of the probe string on the Bañados-Teitelboim-Zanelli black hole [15] given by a special case of the Horndeski theory [16], given by a Lagrangian

$\mathcal{L}=\kappa R-2 \Lambda-\gamma X+\beta G_{\mu \nu} \phi^{\mu} \phi^{\nu}$

where $\kappa, \gamma$ and $\beta$ are coupling constant, $X:=\partial_{\mu} \phi \partial^{\mu} \phi$, $\phi_{\mu}:=\nabla_{\mu} \phi$, while that $R, G_{\mu \nu}$ and $\Lambda$ are the scalar curvature, the Einstein tensor and the cosmological constant respectively. For the theory (1), the inclusion of the fundamental string is given through the NG term. Here, although the string is stationary, provides maximum growth in complexity where the action growth is influenced by the parameters of the theory.

On the other hand, anti-de-Sitter (AdS) planar black holes allow us to analyze the effects on shear viscosity, thanks to the gravity/gauge duality [17-19] and by effective coupling constants of the transverse graviton on the location of the event horizon [20], where the fluid is characterized by the production of entropy [21]. For some common substances (for example helium, nitrogen, and water) the viscosity/entropy density $(\eta / s)$ ratio is always substantially greater than its 
value in dual gravity theories, which reads as [22-24]

$\frac{\eta}{s} \geq \frac{1}{4 \pi}$

known as the Kovtun-Son-Starinets (KSS) bound. This value has been shown in a variety of cases (see [25-28]). However, there are concrete examples where the KSS bound (2) is violated, for example, via gravitational theories such as the five-dimensional Einstein-Hilbert Gauss-Bonnet model $[29,30]$ as well as scalar-tensor theories [21,31], where the Lagrangian is given by (1).

The above leads us to the motivations of the present paper, which is to explore hairy black holes solutions in four dimensions and their thermodynamics, allowing us to obtain the CA conjecture following the procedure performed in $[2,3]$, considering a Wilson line operator located in the $\mathrm{AdS}_{4}$ spacetime, by inserting a fundamental string, described by the addition of the NG term and computing the growth of the complexity of a dissipative system. Together with the above, constructing a Noether charge with a suitable election of a space-like Killing vector, we can compute the $\eta / s$ ratio by using the infrared data on the black hole event horizon [32], greatly simplifying the steps unlike the traditional procedures $[24,30]$. For all these calculations, the action is described by the four-dimensional Degenerate-Higher-Order-ScalarTensor (DHOST) theory

$$
\begin{aligned}
S\left[g_{\mu \nu}, \phi\right] & =\int d^{4} x \sqrt{-g} \mathcal{L}, \\
\mathcal{L} & =Z(X)+G(X) R+\sum_{i=2}^{5} A_{i} \mathcal{L}_{i},
\end{aligned}
$$

where

$$
\begin{aligned}
& \mathcal{L}_{2}:=\left((\square \phi)^{2}-\phi_{\mu \nu} \phi^{\mu \nu}\right), \quad \mathcal{L}_{3}:=\square \phi \phi^{\mu} \phi_{\mu \nu} \phi^{\nu}, \\
& \mathcal{L}_{4}:=\phi^{\mu} \phi_{\mu \nu} \phi^{\nu \rho} \phi_{\rho}, \quad \mathcal{L}_{5}:=\left(\phi^{\mu} \phi_{\mu \nu} \phi^{\nu}\right)^{2},
\end{aligned}
$$

and the equations of motions with respect to the metric $g_{\mu \nu}$ and the scalar field $\phi$ can be written in the following form

$\mathcal{E}_{\mu \nu}:=\mathcal{G}_{\mu \nu}^{Z}+\mathcal{G}_{\mu \nu}^{G}+\sum_{i=2}^{5} \mathcal{G}_{\mu \nu}^{(i)}=0$,

$\varepsilon_{(\phi)}=\nabla_{\mu} \mathcal{J}^{\mu}=\nabla_{\mu}\left[\frac{\delta \mathcal{L}}{\delta\left(\phi_{\mu}\right)}-\nabla_{\nu}\left(\frac{\delta \mathcal{L}}{\delta\left(\phi_{\mu \nu}\right)}\right)\right]=0$,

where $\mathcal{G}_{\mu \nu}^{Z}, \mathcal{G}_{\mu \nu}^{G}$, the $\mathcal{G}_{\mu \nu}^{(i)}$ 's and $J^{\mu}$ are reported in the Appendix. Here we defined $\phi_{\mu \nu}:=\nabla_{\mu} \nabla_{\nu} \phi$.

Recently, the DHOST theory (3a) and (3b) has attracted a lot of attention for many reasons, one of them is the simple way to add new degrees of freedom through the introduction of a scalar field $\phi$, being an extension of the Horndeski theory [16], and avoiding Ostrogradsky instability due to its degeneracy property (see for example $[33,34]$ ), as well as allows constructing regular black hole solutions via a KerrSchild transformation [35,36], rotating stealth black holes with a cohomogeneity -1 metric [37] and three-dimensional spinning configurations [38]. Here is important to note that the functions $Z, G$ and $A_{i}$ for $i=\{2, \ldots, 5\}$, are arbitrary functions of the kinetic term $X$, where for example for $Z(X)=-2 \Lambda, G(X)=\kappa$ and $A_{i}=0 \quad \forall i$, the EinsteinHilbert action together with a cosmological constant $\Lambda$ is recovered, while that for

$A_{i}=0$, with $i=\{3,4,5\}$ and $A_{2}:=-2 G_{X}$,

where $G_{X}:=d G / d X$, we have a subclass of the Horndeski theory [16] which enjoys shift symmetry $\phi \rightarrow \phi+$ constant and $\phi \rightarrow-\phi$, being deeply analyzed in [39]. Just for completeness, the linear case (1) is recovered with (8) and

$Z(X)=-2 \Lambda-\gamma X, \quad G(X)=\kappa-\left(\frac{\beta}{2}\right) X$.

The plan of the paper is organized as follows: in Sect. 2 we present a black hole solution considering the theory ( $3 a$ ) and (3b), analyzing the thermodynamic quantities in Sect. 3. In Sect. 4, we calculate the NG term of the string moving on the black hole. In Sect. 5, the shear viscosity is computed, obtaining the $\eta / s$ ratio and showing its dependence on the coupling functions, where the KSS bound can be violated. Finally, Sect. 6 is devoted to our conclusions and discussions.

\section{A black hole solution in generalized scalar-tensor theories}

For the generalized scalar-tensor model (3a) and (3b), we consider the following four-dimensional metric Ansatz:

$$
\begin{aligned}
d s^{2} & =-h(r) d t^{2}+\frac{d r^{2}}{f(r)}+r^{2} d \Omega_{2, \epsilon}^{2}, \\
\phi(t, r) & =\psi(r)+\phi_{0} t,
\end{aligned}
$$

where

$d \Omega_{2, \epsilon}^{2}= \begin{cases}d x_{1}^{2}+\sin ^{2}\left(x_{1}\right) d x_{2}^{2}, & \text { for } \epsilon=1, \\ d x_{2}^{2}+\sinh ^{2}\left(x_{1}\right) d x_{2}^{2}, & \text { for } \epsilon=-1, \\ d x_{1}^{2}+d x_{2}^{2}, & \text { for } \epsilon=0,\end{cases}$

and $\phi_{0}$ is an integration constant. In order to simplify our computations, given the structure of the metric (10) and the scalar field $\phi$ from (11), we suppose that the kinetic term

$$
X=\partial_{\mu} \phi \partial^{\mu} \phi=g^{t t}\left(\partial_{t} \phi\right)^{2}+g^{r r}\left(\partial_{r} \phi\right)^{2}=-\frac{\phi_{0}^{2}}{h}+f\left(\psi^{\prime}\right)^{2},
$$

is a constant, this is due to the complexity to compute the NG term, which will be studied in Sect. 4. The above implies 
that the square of the derivative of the radial component of the scalar field $\phi$ can be cast as

$$
\left(\psi^{\prime}\right)^{2}=\frac{\phi_{0}^{2}}{h f}+\frac{X}{f},
$$

where $\left({ }^{\prime}\right)$ denotes the derivative respects to the radial coordinate $r$.

In the following lines, to simplify computations and to show in a simple way the solutions, we fix the function $A_{5}$ as [38]

$A_{5}=\frac{\left(2 A_{2}+X A_{3}+4 G_{X}\right)^{2}}{2 X\left(G+X A_{2}\right)}-\frac{A_{3}+A_{4}}{X}$.

Here we note that this relation is not new. In fact, in the literature, we can find gravity theories given by (3a), which enjoy relations between the coupling functions (see for example $[36,37,40-42])$. In what follows, we will consider the Einstein equation (6) and defining the functions

$$
\begin{aligned}
& \mathcal{Z}_{1}=G+X A_{2}, \\
& \mathcal{Z}_{2}=2 A_{2}+X A_{3}+4 G_{X} .
\end{aligned}
$$

Here, the $(r, r)$-component of the Einstein equations (6) together with $\mathcal{J}^{r}=0$ from (7), allow to obtain a relation between the metric functions $f$ and $h$ given by

$f=\frac{X h\left(r^{2} Z+2 \epsilon G\right)}{2\left[X \mathcal{Z}_{1}(h r)^{\prime}-\phi_{0}^{2}\left(G-\mathcal{Z}_{1}\right)\right]}$,

and the $(t, t)$-component of the Einstein equations yields a second order differential equation for the metric function $h$ which reads

$$
\begin{aligned}
& \left\{\mathcal { Z } _ { 1 } \left[r X \mathcal{Z}_{1}\left(r^{2} Z+2 \epsilon G\right) h^{\prime \prime}+4 \epsilon X \mathcal{Z}_{1} G h^{\prime}-2 Z r X \mathcal{Z}_{1} h\right.\right. \\
& \left.\left.\quad-2 Z r \phi_{0}^{2}\left(\mathcal{Z}_{1}-G\right)\right] X h^{2}\right\} /\left\{2 r \left[X \mathcal{Z}_{1}(h r)^{\prime}\right.\right. \\
& \left.\left.\quad-\phi_{0}^{2}\left(G-\mathcal{Z}_{1}\right)\right]^{2}\right\}=0
\end{aligned}
$$

If we suppose $X \neq 0$ and $\mathcal{Z}_{1} \neq 0$, the metric function $h$ takes the form

$h(r)=\left(6 G \epsilon+r^{2} Z\right) C_{0}-\frac{M}{r}+\frac{\phi_{0}^{2}\left(G-\mathcal{Z}_{1}\right)}{X \mathcal{Z}_{1}}$,

with $C_{0}$ and $M$ integrations constants, while that $\mathcal{J}^{t}=0$ from (7) is trivially satisfied. Then, replacing the metric function $h$ from (18) in (16), we have that

$\frac{h}{f}=6 \mathcal{Z}_{1} C_{0}=$ constant

imposing without loss of generality that

$C_{0}=\frac{1}{6 \mathcal{Z}_{1}}$.
Finally the $\left(x_{i}, x_{i}\right)$-component of the Einstein equations yields the expression

$r^{2} \mathcal{G}(X)+\left(\epsilon X+\phi_{0}^{2}\right)=0$,

where

$\mathcal{G}(X)=\frac{X\left[4\left(\mathcal{Z}_{1} Z\right)_{X}-3 Z \mathcal{Z}_{2}\right]}{4\left[2\left(\mathcal{Z}_{1} G\right)_{X}-G \mathcal{Z}_{2}\right]}$

and the solution is given when

$$
\begin{aligned}
h(r)=f(r) & =\epsilon+\frac{Z r^{2}}{6 \mathcal{Z}_{1}}-\frac{M}{r}, \\
\left(\psi^{\prime}\right)^{2} & =\frac{X(f-\epsilon)}{f^{2}}, \quad \mathcal{G}(X)=0=\epsilon X+\phi_{0}^{2},
\end{aligned}
$$

where we suppose that the integration constant $M$, related to the mass black hole $\mathcal{M}$, is always positive. Many commentaries can be made respect to the solution (21)-(24). First that all, the Eq. (21) shows that for the planar base manifold for the event horizon $(\epsilon=0)$ only a radial dependence for the scalar field is needed (this is $\phi_{0}=0$ ). Together with the above, for the other cases, the sign of the kinetic term must be adjusted to have $\phi_{0}^{2}=-\epsilon X>0$. In addition, this solution resembles the four-dimensional metric with the presence of an effective cosmological constant and a non-trivial expression for the scalar field $\phi(t, r)$. These configurations have been explored in [43-45] with the special truncation of the Horndeski theory (9), in [39] with the conditions (8) and in [38] for the three-dimensional situation with the complete action (3a) and (3b). Nevertheless, these studies begin with a kinetic term $X=X(r)$, in our situation, a priori we are supposing that this one is constant.

Given that to analyze the NG-term and the KSS bound the mass as well as the entropy are required quantities, in the following section we will derive the thermodynamic properties.

\section{Thermodynamics of the solution}

Given the steps performed in the previous section, results interesting to explore the thermodynamics of the solution (21)-(24). As a first computation, we will consider the Hawking Temperature $T$ which reads

$$
T=\left.\frac{\kappa}{2 \pi}\right|_{r=r_{h}}=\frac{1}{4 \pi}\left(\frac{3 r_{h}}{L^{2}}+\frac{\epsilon}{r_{h}}\right),
$$

constructed by the surface gravity given by

$\kappa=\sqrt{-\frac{1}{2}\left(\nabla_{\mu} \xi_{v}\right)\left(\nabla^{\mu} \xi^{\nu}\right)}$, 
with a time-like Killing vector $\partial_{t}=\xi^{\mu} \partial_{\mu}$. Here we have defined

$L^{2}=\frac{6 \mathcal{Z}_{1}}{Z}$,

and $r_{h}$ is the location of the event horizon.

On the other hand, the mass of these configurations will be calculated via the quasilocal formulation described in $[46,47]$, corresponding to an off-shell prescription of the Abbott-Deser-Tekin (ADT) [48-50] procedure. This technique has shown to be useful to compute the mass of black hole solutions with non-standard asymptotically behavior [51] and even with quadratic corrections (see for example [52-55]). The principal elements are the surface term $J^{\mu}$ and the Noether potential $Q^{\mu \nu}$, given by [38]

$$
\begin{aligned}
J^{\mu}= & \sqrt{-g}\left[2\left(P^{\mu(\alpha \beta) \gamma} \nabla_{\gamma} \delta g_{\alpha \beta}-\delta g_{\alpha \beta} \nabla_{\gamma} P^{\mu(\alpha \beta) \gamma}\right)\right. \\
& +\frac{\delta \mathcal{L}}{\delta\left(\phi_{\mu}\right)} \delta \phi-\nabla_{\nu}\left(\frac{\delta \mathcal{L}}{\delta\left(\phi_{\mu \nu}\right)}\right) \delta \phi+\frac{\delta \mathcal{L}}{\delta\left(\phi_{\mu \nu}\right)} \delta\left(\phi_{\nu}\right) \\
& -\frac{1}{2} \frac{\delta \mathcal{L}}{\delta\left(\phi_{\mu \rho}\right)} \phi^{\sigma} \delta g_{\sigma \rho}-\frac{1}{2} \frac{\delta \mathcal{L}}{\delta\left(\phi_{\rho \mu}\right)} \phi^{\sigma} \delta g_{\sigma \rho} \\
& \left.+\frac{1}{2} \frac{\delta \mathcal{L}}{\delta\left(\phi_{\sigma \rho}\right)} \phi^{\mu} \delta g_{\sigma \rho}\right], \\
Q^{\mu \nu}= & \sqrt{-g}\left[2 P^{\mu \nu \rho \sigma} \nabla_{\rho} \xi_{\sigma}-4 \xi_{\sigma} \nabla_{\rho} P^{\mu \nu \rho \sigma}+\frac{\delta \mathcal{L}}{\delta \phi_{\mu \sigma}} \phi^{\nu} \xi_{\sigma}\right. \\
& \left.-\frac{\delta \mathcal{L}}{\delta \phi_{\nu \sigma}} \phi^{\mu} \xi_{\sigma}\right],
\end{aligned}
$$

where $P^{\mu \nu \lambda \rho}=\delta \mathcal{L} / \delta R_{\mu \nu \lambda \rho}$, and $\mathcal{L}$ is the Lagrangian given previously in (3b). Finally, by using a parameter $\zeta \in[0,1]$, we interpolate between the solution at $\zeta=1$ and the asymptotic one at $\zeta=0$, obtaining the quasilocal charge

$\mathcal{M}(\xi)=\int_{B} d^{2} x_{\mu \nu}\left(\Delta Q^{\mu \nu}(\xi)-2 \xi^{[\mu} \int_{0}^{1} d \zeta J^{\nu]}\right)$,

with $\Delta Q^{\mu \nu}(\xi):=Q_{\zeta=1}^{\mu \nu}(\xi)-Q_{\zeta=0}^{\mu \nu}(\xi)$. Via a time-like Killing vector $\xi=\partial_{t}$, we have

$\Delta Q^{\mu \nu}(\xi)=\left(-3 G+4 \mathcal{Z}_{1}\right) \Omega_{2, \epsilon}$,

$\int_{0}^{1} d \zeta J^{r}=\left(3 G-2 \mathcal{Z}_{1}\right) M \Omega_{2, \epsilon}$,

and the mass $\mathcal{M}$ acquires the structure

$\mathcal{M}=2 \mathcal{Z}_{1} M \Omega_{2, \epsilon}=2 \mathcal{Z}_{1} \Omega_{2, \epsilon}\left(\frac{r_{h}^{3}}{L^{2}}+\epsilon r_{h}\right)$,

where $\Omega_{2, \epsilon}$ is the finite volume of the 2-dimensional compact angular base manifold.

To compute the entropy, we will consider the Wald procedure $[56,57]$. The steps start with the surface term given previously in (28), defining a 1 -form $J_{(1)}=J_{\mu} d x^{\mu}$ and its corresponding Hodge dual $\Theta_{(3)}=(-1) * J_{(1)}$. Then, with the equations of motions, we have that

$J_{(3)}=\Theta_{(3)}-i_{\xi} * \mathcal{L}=-d * J_{(2)}$,

where $i_{\xi}$ is a contraction of the vector $\xi^{\mu}$ on the first index of $* \mathcal{L}$. The above allows to define a 2-form $Q_{(2)}=* J_{(2)}$ such that $J_{(3)}=d Q_{(2)}$. Finally, taking $\xi^{\mu}$ as a time-like Killing vector that is null on the event horizon, the variation of the Hamiltonian reads

$\delta \mathcal{H}=\delta \int_{\mathcal{C}} J_{(3)}-\int_{\mathcal{C}} d\left(i_{\xi} \Theta_{(3)}\right)=\int_{\Sigma^{(2)}}\left(\delta Q_{(2)}-i_{\xi} \Theta_{(3)}\right)$

where $\mathcal{C}$ and $\Sigma^{(2)}$ are a Cauchy Surface and its boundary respectively, which has two components, located at infinity $\left(\mathcal{H}_{\infty}\right)$ and at the horizon $\left(\mathcal{H}_{+}\right)$. According to Wald formalism, the first law of black holes thermodynamics

$d \mathcal{M}=T d \mathcal{S}_{W}$

is a consequence of $\delta \mathcal{H}_{\infty}=\delta \mathcal{H}_{+}$. With these ingredients, computing the respective variation of the solution (21)-(24), at the infinity

$\delta \mathcal{H}_{\infty}=2 \mathcal{Z}_{1} \Omega_{2, \epsilon} \delta M \Rightarrow \mathcal{H}_{\infty}=2 \mathcal{Z}_{1} \Omega_{2, \epsilon} M$,

being consistent with the expression for the mass computed previously in (32). While that at the horizon with the Hawking temperature (25)

$\delta \mathcal{H}_{+}=\left(\epsilon+\frac{3 r_{h}}{L^{2}}\right) \mathcal{Z}_{1} \Omega_{2, \epsilon} \delta r_{h}=T \delta\left(4 \pi r_{h}^{2} \Omega_{2, \epsilon} \mathcal{Z}_{1}\right)$,

obtaining the entropy of the solution, which reads

$\mathcal{S}_{W}=4 \pi r_{h}^{2} \Omega_{2, \epsilon} \mathcal{Z}_{1}$

It is worth pointing out that for the planar situation $(\epsilon=0)$, besides the fulfills of the first law (35), a four-dimensional Smarr relation [58]

$\mathcal{M}=\frac{2}{3} T \mathcal{S}_{W}$,

is satisfied.

With these thermodynamic quantities, in particular the mass $\mathcal{M}$, we are in a position to analyze the NG term, which will be explored in the following section. 


\section{Evaluating on the NG action}

In this section, we present the NG action to evaluating to the probe string [1-3], which is given by

$S_{N G}=-T_{s} \int d \sigma \sqrt{-\operatorname{detg}_{\text {ind }}}$,

where $T_{s}$ is the fundamental string tension. Adding the Wilson loop implies the insertion of a fundamental string whose world-sheet has a limit in this loop, computing the NG action of this fundamental string. Furthermore, we can calculate the derivative with respect to the time of the NG action, obtained by integrating the square root of the determinant of the induced metric. In order to study the evolution of the complexity, we draw the Penrose diagram of the causal structure of the hairy black holes given by (21)-(24). For this, to describe the null sheets bounding of the WdW path, it is more convenient to define the tortoise coordinate

$r^{*}=\int \frac{d r}{f(r)}, \quad r_{\infty}^{*}=\lim _{r \rightarrow \infty} r^{*}(r)$,

where we can define the Eddington-Finkelstein coordinates $u=t+r^{*}$ and $v=t-r^{*}$, which describe outgoing and ingoing null rays respectively. According to [59], the causal structure of the black holes (23) is illustrated by the Penrose diagram in the Fig. 1, where we are interested in the time dependence of the complexity and therefore in the time dependence of the gravitational action evaluated on this path, as the boundary time increases.

Now, we will present the effect of the string moving on this spacetime geometry, computing the induced metric using the parameters $\tau$ and $\sigma$ in the world-sheet of the fundamental string. These parameters are given as follows

$t=\tau, \quad r=\sigma, \quad \varphi=\omega \tau+\xi(\sigma)$,

where $\omega$ is a constant angular velocity and $\xi(\sigma)$ is a function that determines the string's shape. Considering $d \Omega_{2, \epsilon}^{2}=$ $d \varphi^{2}$, we have that the metric induced in the world-sheet is given by

$$
\begin{aligned}
& d s_{i n d}^{2}=H(\sigma) d \tau^{2}+G(\sigma) d \sigma^{2}+2 F(\sigma) d \tau d \sigma, \\
& H(\sigma)=-f(\sigma)+\sigma^{2} \omega^{2},
\end{aligned}
$$

$$
\begin{aligned}
c_{\xi}= & \sigma_{H}^{2} \omega, \\
\sigma_{H}= & -\frac{(2 / 3)^{1 / 3} \epsilon L}{\left[9\left(1-v^{2}\right)(M / L)+\sqrt{3} \sqrt{27\left(1-v^{2}\right)^{4}(M / L)^{2}-4 \epsilon^{3}\left(1-v^{2}\right)^{3}}\right]^{1 / 3}} \\
& +\frac{L\left[9\left(1-v^{2}\right)(M / L)+\sqrt{3} \sqrt{27\left(1-v^{2}\right)^{4}(M / L)^{2}-4 \epsilon^{3}\left(1-v^{2}\right)^{3}}\right]^{1 / 3}}{2^{1 / 3}(3)^{2 / 3}},
\end{aligned}
$$


Fig. 1 Penrose diagram of an eternal black hole. In the WdW patch, this black hole moves forward in time in a symmetric way $\left(t_{L}=t_{R}\right)$. For both cases, we can see that Region I is the zone behind the future horizon; Region II corresponds to the sector outside both horizons, and Region III is located behind the past horizon
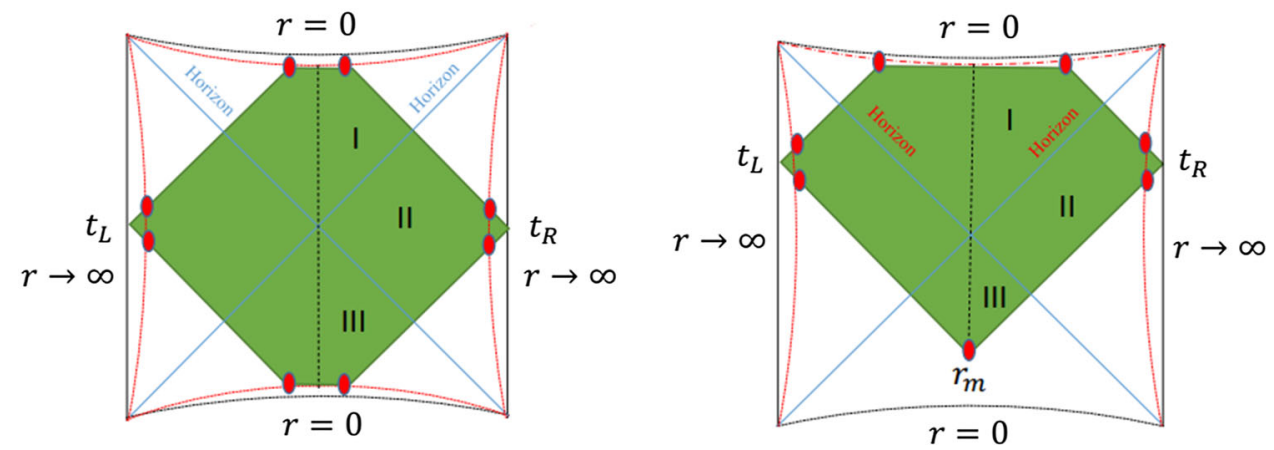

where $\sigma=\sigma_{H}$ is the solution for numerator of the square root in Eq. (49), and $v=\omega L$ is the angular velocity. We can observe that $\mathcal{L}=T_{S} \sigma \xi^{\prime} f(\sigma) / c \xi$, and we can write

$$
\begin{aligned}
\frac{d S_{N G}}{T_{s} d t}= & \int_{0}^{r_{h}} d \sigma \sqrt{\frac{\sigma\left[\left(\sigma^{2}+\sigma \sigma_{H}+\sigma_{H}^{2}\right)\left(1-v^{2}\right)+\epsilon L^{2}\right]}{\sigma^{3}+\sigma^{2} \sigma_{H}+\left(\sigma_{H}^{2}+\epsilon L^{2}\right) \sigma+\sigma_{H}^{3} v^{2}}} \\
r_{h}= & -\frac{(2 / 3)^{1 / 3} \epsilon L}{\left[9(M / L)+\sqrt{3} \sqrt{27(M / L)^{2}+4 \epsilon^{3}}\right]^{1 / 3}} \\
& +\frac{L\left[9(M / L)+\sqrt{3} \sqrt{27(M / L)^{2}+4 \epsilon^{3}}\right]^{1 / 3}}{2^{1 / 3}(3)^{2 / 3}}
\end{aligned}
$$

As we can see, this integral is similar to the solutions explored in [3]. Nevertheless, is interesting to note that in (52) the action growth depends on the mass of the black hole (32) as well as the angular velocity (which depends of $L$ from (27)), where the functions $\mathcal{Z}_{1}(X)$ and $Z(X)$ are present. This shows the importance of the coupling functions given by the DHOST theory (3a) and (3b). Together with the above, as in [3], we perform numerical procedures to solve the integral (52) and (53), being reflected in the Figs. 2, 3 and 4 for the three topologies, with $\epsilon=1, \epsilon=0$, and $\epsilon=-1$ respectively.

According to Fig. 2, from the top panel, we can see that the growth of the action is larger as the mass increases and the string moves slower, while that when the string moves faster, the effect becomes less, showing us the effect of the drag force. In addition, from the bottom panel we have that when the angular velocity is small, the growth action is an increasing function of the mass. Nevertheless, for some special value of $v$ near to one, becomes decreasing.

For the planar case, as shown in Fig. 3, we have that the action growth versus angular velocity (top panel) resemble the spherical case. In addition, the action growth versus $M$ (bottom panel) provides us that always is an increasing function.

Figure 4 shows that for the hyperbolic case we have that the action growth for regions of great mass corresponds to the region where the string moves more slowly (top panel).
Nevertheless, from the bottom panel, we note that exists some values of the angular velocity, near to one, where the action growth is simultaneously a decreasing and then an increasing function of $M$, unlike the spherical or planar situation. We can also note that for this case, the causal structure of the black hole has two horizons, one external and one internal. Nevertheless, as was explained in Sect. 2, we are focused on the case with $r_{h}>L$, where the mass $\mathcal{M}$ is always positive.

In resume, and according to the $\mathrm{CA}$ conjecture where the action growth is equal to the complexity growth, such growth of the complexity for the three cases are controlled by coupling functions present in the DHOST theory (3a)(5), where we can see that this growth is larger as the mass increases and the string moves slower (top panel of Figs. 2, 3, 4) . This result is consistent concerning the analysis performed in discussions of [3], where now for the planar or spherical case $(\epsilon \in\{0,1\})$, we have that the growth of the complexity is an increasing function of the mass when the angular velocity is small, unlike the hyperbolical situation $(\epsilon=-1)$, where we can find some values where the action growth is simultaneously a decreasing and then an increasing function of $M$, as shown in the bottom panel of Figs. 2, 3 and 4. Additionally, in general, we have that the velocity dependence of the stationary string gives the maximal complexity growth, where the growth of the complexity decreases as the probe string moves faster.

On the other hand, althought the above analysis is similar to the cases studied in [2,3], in Figs. 2, 3 and 4 we impose that the radius $L$ takes a particular value, but this parameter as well as the mass depend on the functions $Z$ and $\mathcal{Z}_{1}$. In fact, if we suppose that for the spherical case the mass $M=\mathcal{M} /\left(2 \mathcal{Z}_{1} \Omega_{2,1}\right)$ is fixed, yet we can change the values of $L$ from (27) with a suitable election of the coupling functions satisfy (21), given in the Fig. 5. The above shows us the importance of the coupling functions from the DHOST theory (3a)-(5).

In the following section, we will explore the shear viscosity for the planar hairy configuration (this is, the solution (21)-(24) with $\epsilon=0$ ), where the thermodynamical quantity $\mathcal{S}_{W}$ computed previously in (38) has a providential role. 
Fig. 2 Graphics of the action growth versus angular velocity $v$ and the mass dependence $M=\mathcal{M} /\left(2 \mathcal{Z}_{1} \Omega_{2,1}\right)$ with $\epsilon=1$. Top panel: Action growth for the hairy Black hole (23) and (24) and angular velocity dependence $v$ for the values: $M=0.1, M=0.2, M=0.3$, $M=0.4, M=0.5 M=0.6$. Bottom panel: Action growth for the hairy Black hole (23) and (24) with for the values: $v=0.3, v=0.4, v=0.5$, $v=0.6, v=0.7, v=0.8$. For both cases, we impose $L=0.01$ and $\Omega_{2,1}=1$

\section{The viscosity/entropy density ratio}

As was shown in the introduction, AdS-planar black hole configurations allows us to study the viscosity/entropy $\operatorname{density}(\eta / s)$ ratio. Just to clarify, in the following computations we impose $\epsilon=0$ from the thermodynamical quantities and the solution found in (21)-(24). As a first step, we perform a transverse and traceless perturbation on the metric (10) with $h=f$, which reads

$d s^{2}=-f(r) d t^{2}+\frac{d r^{2}}{f(r)}+2 r^{2} \Psi(t, r) d x_{1} d x_{2}$
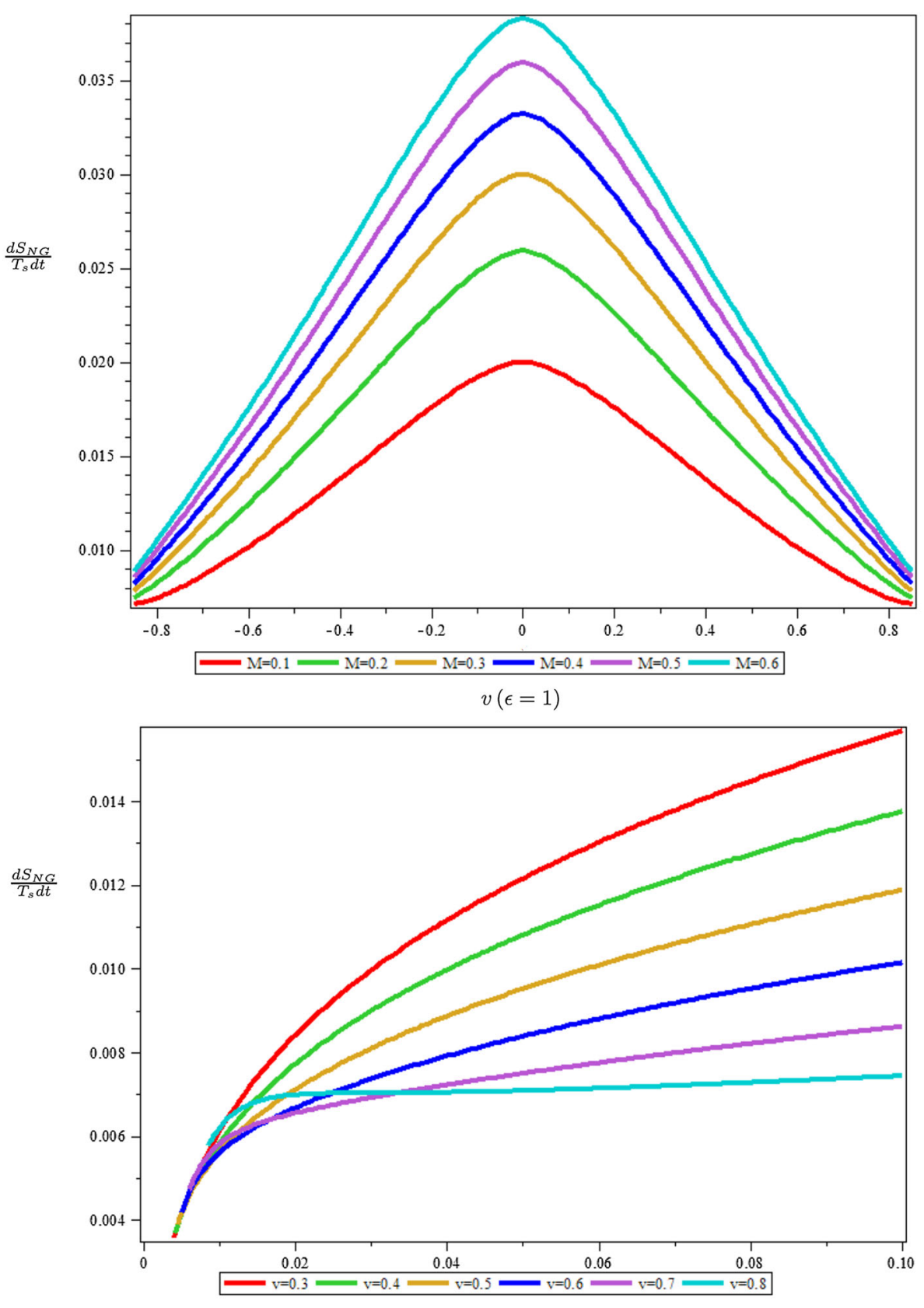

$M(\epsilon=1)$

$+r^{2}\left(d x_{1}^{2}+d x_{2}^{2}\right)$,

where with the Ansatz

$\Psi(t, r)=\gamma t+h_{x_{1} x_{2}}(r)$,

with $\gamma$ a constant identified as the gradient of the fluid velocity along the $x_{1}$ direction [32], yields the following linearized equations for $h_{x_{1} x_{2}}$ :

$\left[\mathcal{Z}_{1} r^{2} f\left(h_{x_{1} x_{2}}\right)^{\prime}\right]^{\prime}=0$ 
Fig. 3 Graphics of the action growth versus angular velocity $v$ and the mass dependence $M=\mathcal{M} /\left(2 \mathcal{Z}_{1} \Omega_{2,0}\right)$ with $\epsilon=0$. Top panel: action growth for the hairy Black hole (23) and (24) and angular velocity dependence $v$ for the values: $M=0.1, M=0.2, M=0.3$, $M=0.4, M=0.5 M=0.6$. Bottom panel: action growth for the hairy Black hole (23) and (24) with for the values: $v=0.3, v=0.4, v=0.5$, $v=0.6, v=0.7, v=0.8$. For both cases, we impose $L=0.01$ and $\Omega_{2,0}=1$
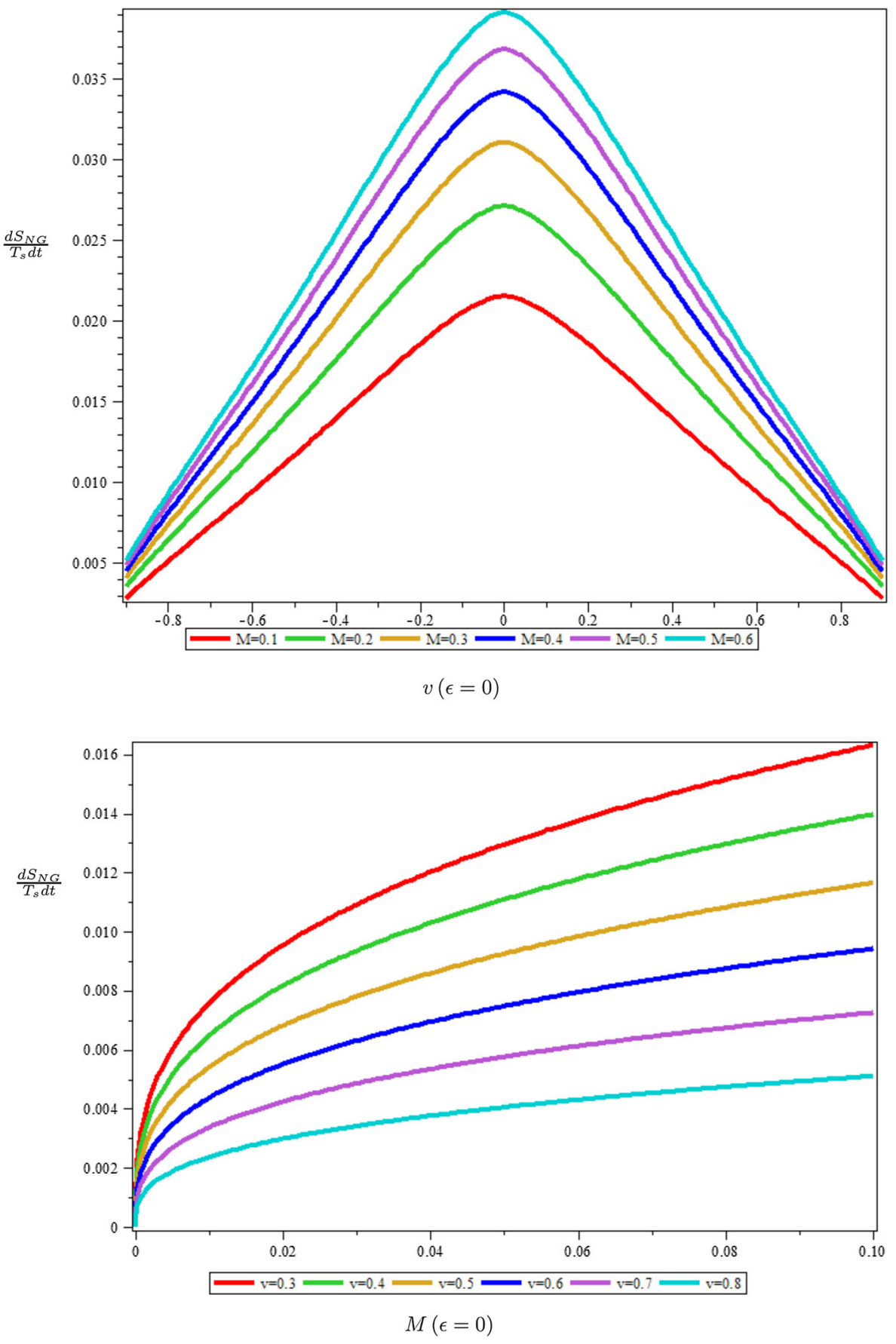

According to the Wald formalism [56,57] together with the method [32], the shear stress is associated to the current

$\mathcal{J}^{x_{2}}=\sqrt{-g} Q^{r x_{2}}=\mathcal{Z}_{1} r^{2} f\left(h_{x_{1} x_{2}}\right)^{\prime}$,

which is conserved due to $\left(\mathcal{J}^{x_{2}}\right)^{\prime}$ is the linear equation (56), constructed with the Noether potential $\mathcal{Q}^{\mu v}$ given previously in (29) and a space-like Killing vector $\partial_{x_{1}}=\xi^{\mu} \partial_{\mu}$. Imposing the ingoing horizon boundary condition

$h_{x_{1} x_{2}}=\gamma \sqrt{\frac{G}{\mathcal{Z}_{1}}} \frac{\log \left(r-r_{h}\right)}{4 \pi T}+\cdots$,

as well as a Taylor expansion in the near horizon region $r_{h}$

$h=f=4 \pi T\left(r-r_{h}\right)+\cdots$, 
Fig. 4 Graphics of the action growth versus angular velocity $v$ and the mass dependence $M=\mathcal{M} /\left(2 \mathcal{Z}_{1} \Omega_{2,-1}\right)$ with $\epsilon=-1$. Top panel: action growth for the hairy Black hole (23) and (24) with angular velocity dependence $v$ for the values: $M=0.1, M=0.2$, $M=0.3, M=0.4, M=0.5$ $M=0.6$. Bottom panel: action growth for the hairy Black hole (23) and (24) with for the values: $v=0.3, v=0.4, v=0.5$, $v=0.6, v=0.7, v=0.8$. For both cases, we impose $L=0.01$ and $\Omega_{2,-1}=1$
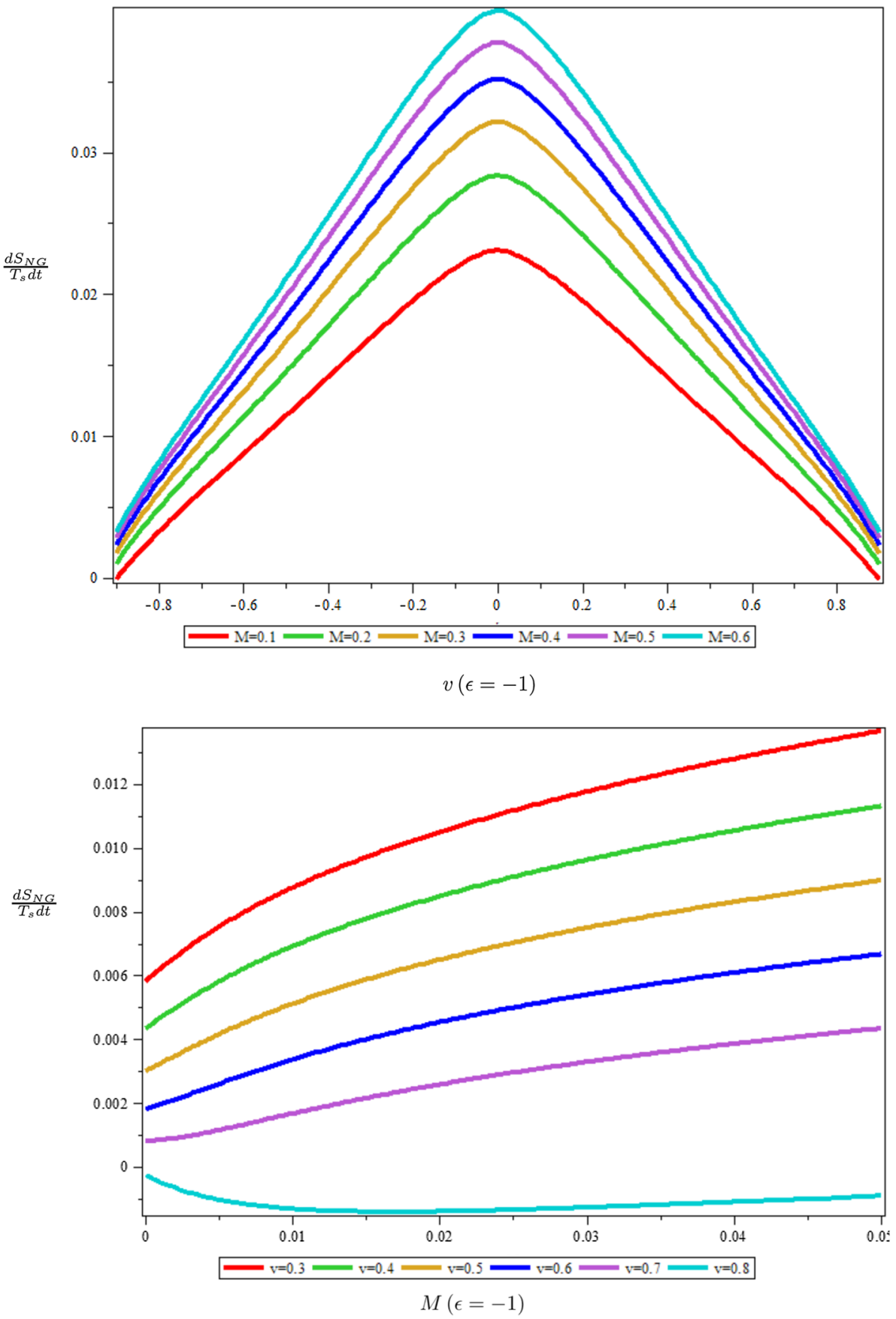

where $T$ is the Hawking temperature (25), we have:

$\eta=\gamma \mathcal{Z}_{1} \sqrt{\frac{G}{\mathcal{Z}_{1}}} r_{h}^{2}=\frac{1}{4 \pi} \sqrt{\frac{G}{\mathcal{Z}_{1}}} \gamma s$,

with $s$ corresponding to the entropy density given by

$s=\frac{\mathcal{S}_{W}}{\Omega_{2,0}}=4 \pi r_{h}^{2} \mathcal{Z}_{1}$.
Finally, the viscosity/entropy density ratio takes the form

$\frac{\eta}{s}=\frac{1}{4 \pi} \sqrt{\frac{G}{\mathcal{Z}_{1}}}=\frac{1}{4 \pi} \sqrt{\frac{G}{G+X A_{2}}}$.

Note that, as the examples computed in [25-28], there is not a presence of the location of the event horizon $r_{h}$ on the $\eta / s$ ratio (62), where for a suitable election of the kinetic term, the ratio goes back to the universal value $1 /(4 \pi)$. For example, in addition to the condition (21), it is possible to find a $X$ such that $G=\mathcal{Z}_{1}$ (see for example the case $G=\kappa, Z=$ 
Fig. 5 Graphics of the action growth versus angular velocity $v$ and a fixed mass with $\epsilon=1$,

where $M=0.1$ and for values of the radius $L: L=0.01$, $L=0.012, L=0.013$

$L=0.014, L=0.015$

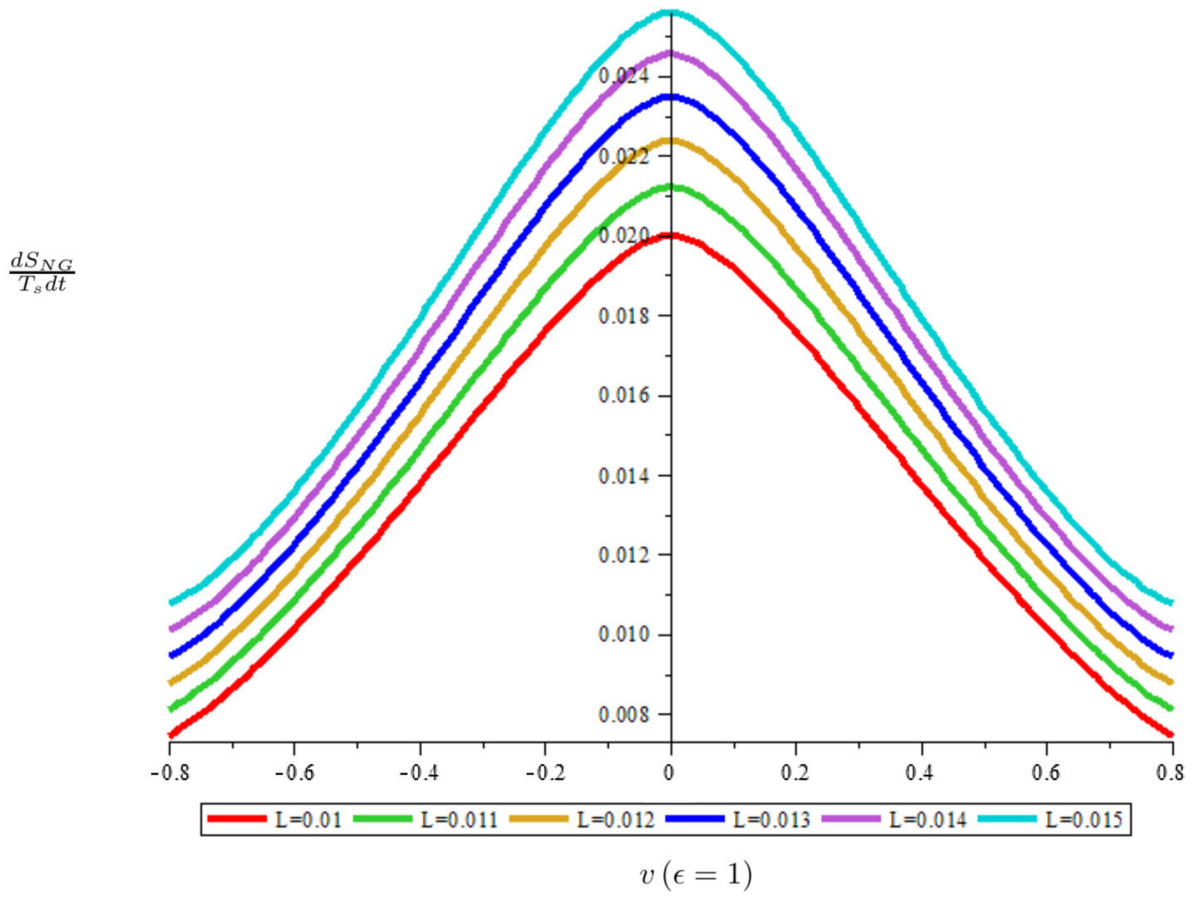

$-2 \Lambda-\gamma X$ and $A_{2}=0$ ), where the KKS bound is satisfied. In fact, the Einstein-Hilbert action together with a cosmological constant is recovered from this case. Nevertheless, in (62) appears a contribution depending on the functions $G$ and $\mathcal{Z}_{1}$, where the four dimensional viscosity/entropy density ratio for special cases studied in [31,60] are recovered, violating the KSS bound. Together with the above, the $\eta / s$ ratio (62) allows us to construct examples where the kinetic term $X$ satisfies (21) and (22) and

$0<\frac{G}{\mathcal{Z}_{1}}=\frac{G}{G+X A_{2}}<1$,

with a suitable choice of coupling functions. For example, for

$G(X)=\kappa+k X^{j}, \quad A_{2}=G_{X}=k j X^{j-1}$,

where $\kappa, X, k$ and $j$ are positive constants, while that $Z$ and $\mathcal{Z}_{2}$ satisfy (21), we have

$0<\frac{G}{\mathcal{Z}_{1}}=\frac{\kappa+k X^{j}}{\kappa+(1+j) k X^{j}}<1$,

implying that

$0<\frac{\eta}{s}<\frac{1}{4 \pi}$

Just for completeness, the viscosity/ entropy density ratio (62) also can be found following the steps described in [24, 30].

\section{Conclusions and discussions}

The aims of the present work were, first, to show that generalized scalar-tensor theories present in (3a) and (3b) admit hairy black holes. Indeed, for this model, the solution resembles the four-dimensional metric with the presence of an effective cosmological constant and a non-trivial expression for the scalar field, with three different topologies, where we are supposing that the kinetic term is a constant. With this result, we exhibit a variety of thermodynamical behaviors, via the quasilocal method and Wald formalism, showing that the first law of black holes thermodynamics holds (35), together with a Smarr relation for the planar situation (39).

With the above, our second motivation was to compute the Nambu-Goto action, obtaining the relation between the action growth and the angular velocity as well as the mass of these solutions, showing different behaviors depending on the topology of the event horizon, presented in Figs. 2, 3 and 4, where according to CA conjecture, these situations represent the growth of the black-hole complexity. Additionally, we note the importance of these analyses of the coupling functions $\mathcal{Z}_{1}(X)$ and $Z(X)$, present in this generalized scalar-tensor model.

As a final aim, for the planar case, we explored the viscosity/entropy density ratio, constructing a conserved charge via the Wald formalism, and a suitable election of the Killing vector, obtaining that this ratio depends on the coupling functions from the theory $\left(G\right.$ and $\left.\mathcal{Z}_{1}\right)$. Here, some particular cases where the KSS bound is not fulfilled were studied. To our knowledge, together with [21,31], our work provides a new 
example of the violation of the $\eta / s$ ratio whose Lagrangian is at most linear in curvature tensor.

Further works are, naturally, to analyze hairy black holes where the kinetic term $X$ is not a constant, higher dimensional configurations and even to study the CA conjecture by using the procedure elaborated in [61], giving a deep discussion of the boundary terms required in the action functional, having an attention to the case of null boundary segments, needing the introduction to associated joints contributions.

Acknowledgements FS would like to thank CNPq and CAPES for partial financial support. FS also would like to thank Komeil Babaei Velni, Yue-Zhou Li, Dmitry Ageev, and Kumar Shwetketu Virbhadra for valuable comments and discussions. The authors thank the Referee for the commentaries and suggestions to improve the paper. MB is supported by PROYECTO INTERNO UCM 2022, LíNEA REGULAR.

Data Availability Statement This manuscript has no associated data or the data will not be deposited. [Authors' comment: This is a theoretical work and no experimental data were used.]

Open Access This article is licensed under a Creative Commons Attribution 4.0 International License, which permits use, sharing, adaptation, distribution and reproduction in any medium or format, as long as you give appropriate credit to the original author(s) and the source, provide a link to the Creative Commons licence, and indicate if changes were made. The images or other third party material in this article are included in the article's Creative Commons licence, unless indicated otherwise in a credit line to the material. If material is not included in the article's Creative Commons licence and your intended use is not permitted by statutory regulation or exceeds the permitted use, you will need to obtain permission directly from the copyright holder. To view a copy of this licence, visit http://creativecomm ons.org/licenses/by/4.0/.

Funded by SCOAP ${ }^{3}$.

\section{Appendix}

Here we report the expressions for $\mathcal{G}_{\mu \nu}^{Z}, \mathcal{G}_{\mu \nu}^{G}$, the $\mathcal{G}_{\mu \nu}^{(i)}$ 's and $\mathcal{J}^{\mu}$ present in the equations of motions (6) and (7)

$$
\begin{aligned}
\mathcal{G}_{\mu \nu}^{Z}= & -\frac{1}{2} Z(X) g_{\mu \nu}+Z_{X} \phi_{\mu} \phi_{\nu}, \\
\mathcal{G}_{\mu \nu}^{G}= & G G_{\mu \nu}+G_{X} R \phi_{\mu} \phi_{\nu}-\nabla_{\nu} \nabla_{\mu} G \\
& +g_{\mu \nu} \nabla_{\lambda} \nabla^{\lambda} G, \\
\mathcal{G}_{\mu \nu}^{(2)}= & -\phi_{\mu}\left(A_{2 X} \nabla_{\nu} X\right) \square \phi-\left(A_{2 X} \nabla_{\mu} X\right) \phi_{\nu} \square \phi \\
& -A_{2} \phi_{\nu \mu} \square \phi-\phi_{\nu \mu} \phi_{\lambda}\left(A_{2 X} \nabla^{\lambda} X\right) \\
& +\phi_{\nu} \phi_{\lambda \mu}\left(A_{2 X} \nabla^{\lambda} X\right)+\phi_{\mu} \phi_{\lambda \nu}\left(A_{2 X} \nabla^{\lambda} X\right) \\
& +A_{2} R_{\nu \lambda} \phi_{\mu} \phi^{\lambda}+A_{2} R_{\mu \lambda} \phi_{\nu} \phi^{\lambda} \\
& -A_{2} \phi_{\lambda \nu \mu} \phi^{\lambda}+\frac{1}{2} A_{2} g_{\mu \nu}(\square \phi)^{2} \\
& +g_{\mu \nu} \phi_{\lambda}\left(A_{2 X} \nabla^{\lambda} X\right) \square \phi+A_{2} g_{\mu \nu} \phi^{\lambda} \phi_{\rho \lambda}^{\rho}
\end{aligned}
$$

$$
\begin{aligned}
& -A_{2} g_{\mu \nu} R_{\lambda \rho} \phi^{\lambda} \phi^{\rho}+\frac{1}{2} A_{2} g_{\mu \nu} \phi_{\rho \lambda} \phi^{\rho \lambda} \\
& +A_{2 X} \phi_{\mu} \phi_{\nu}\left((\square \phi)^{2}-\phi_{\lambda \rho} \phi^{\lambda \rho}\right) \text {, } \\
& \mathcal{G}_{\mu \nu}^{(3)}=-\frac{1}{2} A_{3} \phi_{\mu} \phi_{\nu}(\square \phi)^{2}-\frac{1}{2} \phi_{\mu} \phi_{\nu} \phi_{\lambda}\left(A_{3 X} \nabla^{\lambda} X\right) \square \phi \\
& +\frac{1}{2} A_{3} \phi_{\mu} \phi_{\lambda \nu} \phi^{\lambda} \square \phi+\frac{1}{2} A_{3} \phi_{\nu} \phi_{\lambda \mu} \phi^{\lambda} \square \phi \\
& -\frac{1}{2} A_{3} \phi_{\mu} \phi_{\nu} \phi^{\lambda} \phi_{\rho \lambda}^{\rho}+\frac{1}{2} A_{3} R_{\lambda \rho} \phi_{\mu} \phi_{\nu} \phi^{\lambda} \phi^{\rho} \\
& -\frac{1}{2} \phi_{\mu}\left(A_{3 X} \nabla_{\nu} X\right) \phi^{\lambda} \phi_{\rho \lambda} \phi^{\rho} \\
& -\frac{1}{2}\left(A_{3 X} \nabla_{\mu} X\right) \phi_{\nu} \phi^{\lambda} \phi_{\rho \lambda} \phi^{\rho} \\
& -\frac{1}{2} A_{3} \phi_{\nu} \phi^{\lambda} \phi_{\rho \lambda \mu} \phi^{\rho}-\frac{1}{2} A_{3} \phi_{\mu} \phi^{\lambda} \phi_{\rho \lambda \nu} \phi^{\rho} \\
& -A_{3} \phi_{\nu} \phi^{\lambda} \phi_{\rho \lambda} \phi^{\rho}{ }_{\mu}-A_{3} \phi_{\mu} \phi^{\lambda} \phi_{\rho \lambda} \phi^{\rho}{ }_{\nu} \\
& +\frac{1}{2} g_{\mu \nu} \phi_{\lambda}\left(A_{3 X} \nabla^{\lambda} X\right) \phi^{\rho} \phi_{\sigma \rho} \phi^{\sigma} \\
& +\frac{1}{2} g_{\mu \nu} A_{3} \phi^{\lambda} \phi^{\rho} \phi_{\sigma \rho \lambda} \phi^{\sigma}+g_{\mu \nu} A_{3} \phi^{\lambda} \phi^{\rho} \phi_{\sigma \rho} \phi_{\lambda}^{\sigma} \\
& +A_{3 X} \phi_{\mu} \phi_{\nu}(\square \phi) \phi^{\rho} \phi_{\sigma \rho} \phi^{\sigma} \text {, } \\
& \begin{aligned}
\mathcal{G}_{\mu \nu}^{(4)}= & -A_{4} \phi_{\mu} \phi_{\nu} \phi^{\lambda} \phi_{\rho \lambda}^{\rho}+A_{4} \phi_{\lambda \mu} \phi^{\lambda} \phi_{\rho \nu} \phi^{\rho} \\
& -\phi_{\mu} \phi_{\nu}\left(A_{4 X} \nabla^{\lambda} X\right) \phi_{\rho \lambda} \phi^{\rho}-A_{4} \phi_{\mu} \phi_{\nu} \phi_{\rho \lambda} \phi^{\rho \lambda} \\
& -\frac{1}{2} A_{4} g_{\mu \nu} \phi^{\lambda} \phi^{\rho} \phi_{\sigma \rho} \phi_{\lambda}^{\sigma}+A_{4 X} \phi_{\mu} \phi_{\nu} \phi_{\lambda \rho} \phi^{\lambda} \phi^{\rho \sigma} \phi_{\sigma},
\end{aligned} \\
& \mathcal{G}_{\mu \nu}^{(5)}=-A_{5} \phi_{\mu} \phi_{\nu} \phi^{\lambda} \phi_{\rho \lambda} \phi^{\rho}(\square \phi) \\
& -\phi_{\mu} \phi_{\nu} \phi_{\lambda}\left(A_{5 X} \nabla^{\lambda} X\right) \phi^{\rho} \phi_{\sigma \rho} \phi^{\sigma} \\
& +A_{5} \phi_{\nu} \phi_{\lambda \mu} \phi^{\lambda} \phi^{\rho} \phi_{\sigma \rho} \phi^{\sigma}+A_{5} \phi_{\mu} \phi_{\lambda \nu} \phi^{\lambda} \phi^{\rho} \phi_{\sigma \rho} \phi^{\sigma} \\
& -A_{5} \phi_{\mu} \phi_{\nu} \phi^{\lambda} \phi^{\rho} \phi_{\sigma \rho \lambda} \phi^{\sigma}-2 A_{5} \phi_{\mu} \phi_{\nu} \phi^{\lambda} \phi^{\rho} \phi_{\sigma \rho} \phi_{\lambda}^{\sigma} \\
& -\frac{1}{2} A_{5} g_{\mu \nu} \phi^{\lambda} \phi_{\rho \lambda} \phi^{\rho} \phi^{\sigma} \phi_{\tau \sigma} \phi^{\tau} \\
& +A_{5 X} \phi_{\mu} \phi_{\nu} \phi^{\lambda} \phi^{\rho} \phi_{\rho \lambda} \phi^{\sigma} \phi^{\tau} \phi_{\tau \sigma}
\end{aligned}
$$

while that

$\mathcal{J}^{\mu}=\mathcal{J}_{Z}^{\mu}+\mathcal{J}_{G}^{\mu}+\sum_{i=2}^{5} \mathcal{J}_{(i)}^{\mu}$,

with

$$
\begin{aligned}
\mathcal{J}_{Z}^{\mu}= & 2 Z_{X} \phi^{\mu}, \\
\mathcal{J}_{G}^{\mu}= & 2 G_{X} R \phi^{\mu}, \\
\mathcal{J}_{(2)}^{\mu}= & 2 A_{2 X} \phi^{\mu}\left[(\square \phi)^{2}-\phi_{\lambda \rho} \phi^{\lambda \rho}\right] \\
& -2 \nabla_{\nu}\left[A_{2}\left(g^{\mu \nu}-\phi^{\mu \nu}\right)\right],
\end{aligned}
$$




$$
\begin{aligned}
\mathcal{J}_{(3)}^{\mu}= & 2 A_{3 X} \phi^{\mu} \square \phi \phi^{\lambda} \phi_{\lambda \rho} \phi^{\rho}+2 A_{3} \square \phi \phi_{\lambda}^{\mu} \phi^{\lambda} \\
& -\nabla_{\nu}\left[A_{3}\left(g^{\mu \nu} \phi^{\lambda} \phi_{\lambda \rho} \phi^{\rho}+\square \phi \phi^{\mu} \phi^{\nu}\right)\right], \\
\mathcal{J}_{(4)}^{\mu}= & 2 A_{4 X} \phi^{\mu} \phi^{\sigma} \phi_{\sigma \rho} \phi^{\rho \lambda} \phi_{\lambda}+A_{4}(X)\left[\phi_{\rho}^{\mu} \phi^{\rho \lambda} \phi_{\lambda}\right. \\
& \left.+\phi^{\sigma} \phi_{\sigma \rho} \phi^{\rho \mu}\right]-\nabla_{\nu}\left[A _ { 4 } ( X ) \left(\phi^{\mu} \phi^{\nu \rho} \phi_{\rho}\right.\right. \\
& \left.\left.+\phi^{\sigma} \phi_{\sigma}^{\mu} \phi^{\nu}\right)\right], \\
\mathcal{J}_{(5)}^{\mu}= & 2 A_{5 X} \phi^{\mu}\left(\phi^{\sigma} \phi_{\sigma \rho} \phi^{\rho}\right)^{2}+2 A_{5}(X)\left(\phi^{\sigma} \phi_{\sigma \rho} \phi^{\rho}\right)\left(\phi^{\mu \sigma} \phi_{\sigma}\right. \\
& \left.+\phi^{\sigma \mu} \phi_{\sigma}\right)-2 \nabla_{\nu}\left[A_{5}(X) \phi^{\sigma} \phi_{\sigma \rho} \phi^{\rho} \phi^{\mu} \phi^{\nu}\right] .
\end{aligned}
$$

\section{References}

1. F.F. Santos, Rotating black hole with a probe string in Horndeski gravity. Eur. Phys. J. Plus 135(10), 810 (2020). arXiv:2005.10983 [hep-th]

2. K. Nagasaki, Complexity of $\mathrm{AdS}_{5}$ black holes with a rotating string. Phys. Rev. D 96(12), 126018 (2017). arXiv:1707.08376 [hep-th]

3. K. Nagasaki, Complexity growth of rotating black holes with a probe string. Phys. Rev. D 98(12), 126014 (2018). arXiv: 1807.01088 [hep-th]

4. D.A. Roberts, B. Yoshida, Chaos and complexity by design. JHEP 04, 121 (2017). arXiv:1610.04903 [quant-ph]

5. D. Stanford, L. Susskind, Complexity and shock wave geometries. Phys. Rev. D 90(12), 126007 (2014). arXiv:1406.2678 [hep-th]

6. D. Carmi, S. Chapman, H. Marrochio, R.C. Myers, S. Sugishita, On the time dependence of holographic complexity. JHEP 11, 188 (2017). arXiv:1709.10184 [hep-th]

7. L. Susskind, Computational complexity and black hole horizons. Fortsch. Phys. 64, 24-43 (2016). arXiv:1403.5695 [hep-th]

8. J. Watrous (2008) Quantum computational complexity. arXiv:quant-ph/0804.3401

9. A.R. Brown, L. Susskind, Second law of quantum complexity. Phys. Rev. D 97(8), 086015 (2018). arXiv:1701.01107 [hep-th]

10. A. Almheiri, D. Marolf, J. Polchinski, J. Sully, Black holes: complementarity or firewalls? JHEP 02, 062 (2013). arXiv:1207.3123 [hep-th]

11. D. Harlow, P. Hayden, Quantum computation vs firewalls. JHEP 06, 085 (2013). arXiv:1301.4504 [hep-th]

12. L. Susskind, The typical-state paradox: diagnosing horizons with complexity. Fortsch. Phys. 64, 84-91 (2016). arXiv:1507.02287 [hep-th]

13. A.R. Brown, D.A. Roberts, L. Susskind, B. Swingle, Y. Zhao, Holographic complexity equals bulk action? Phys. Rev. Lett. 116(19), 191301 (2016). arXiv:1509.07876 [hep-th]

14. A.R. Brown, D.A. Roberts, L. Susskind, B. Swingle, Y.Zhao, Complexity, action, and black holes. Phys. Rev. D 93(8), 086006 (2016). arXiv:1512.04993 [hep-th]

15. M. Banados, C. Teitelboim, J. Zanelli, Phys. Rev. Lett. 69, 1849-1851 (1992). https://doi.org/10.1103/PhysRevLett.69.1849 arXiv:hep-th/9204099

16. G.W. Horndeski, Second-order scalar-tensor field equations in a four-dimensional space. Int. J. Theor. Phys. 10, 363 (1974)

17. J.M. Maldacena, Adv. Theor. Math. Phys. 2, 231-252 (1998). https://doi.org/10.1023/A:1026654312961 arXiv:hep-th/9711200

18. S.S. Gubser, I.R. Klebanov, A.M. Polyakov, Phys. Lett. B 428, 105-114 (1998). https://doi.org/10.1016/S0370-2693(98)00377-3 arXiv:hep-th/9802109
19. E. Witten, Adv. Theor. Math. Phys. 2, 253-291 (1998). https://doi. org/10.4310/ATMP.1998.v2.n2.a2 arXiv:hep-th/9802150

20. N. Iqbal, H. Liu, Phys. Rev. D 79, 025023 (2009). https://doi.org/ 10.1103/PhysRevD.79.025023 arXiv:0809.3808 [hep-th]

21. F.A. Brito, F.F. Santos, Black brane in asymptotically Lifshitz spacetime and viscosity/entropy ratios in Horndeski gravity. EPL 129(5), 50003 (2020). arXiv: 1901.06770 [hep-th]

22. P. Kovtun, D.T. Son, A.O. Starinets, Holography and hydrodynamics: diffusion on stretched horizons. JHEP 0310, 064 (2003). arXiv:hep-th/0309213

23. P. Kovtun, D.T. Son, A.O. Starinets, Viscosity in strongly interacting quantum field theories from black hole physics. Phys. Rev. Lett. 94, 111601 (2005). arXiv:hep-th/0405231

24. D.T. Son, A.O. Starinets, JHEP 09, 042 (2002). https://doi.org/10. 1088/1126-6708/2002/09/042 arXiv:hep-th/0205051

25. A. Buchel, J.T. Liu, Phys. Rev. Lett. 93, 090602 (2004). https:// doi.org/10.1103/PhysRevLett.93.090602 arXiv:hep-th/0311175

26. A. Buchel, Phys. Lett. B 609, 392-401 (2005). https://doi.org/10. 1016/j.physletb.2005.01.052 arXiv:hep-th/0408095

27. P. Benincasa, A. Buchel, R. Naryshkin, Phys. Lett. B 645, 309-313 (2007). https://doi.org/10.1016/j.physletb.2006.12.030 arXiv:hep-th/0610145

28. K. Landsteiner, J. Mas, JHEP 07, 088 (2007). https://doi.org/10. 1088/1126-6708/2007/07/088 arXiv:0706.0411 [hep-th]

29. Y. Kats, P. Petrov, JHEP 01, 044 (2009). https://doi.org/10.1088/ 1126-6708/2009/01/044 arXiv:0712.0743 [hep-th]

30. M. Brigante, H. Liu, R.C. Myers, S. Shenker, S. Yaida, Phys. Rev. D 77, 126006 (2008). https://doi.org/10.1103/PhysRevD.77.126006 arXiv:0712.0805 [hep-th]

31. X.H. Feng, H.S. Liu, H. Lü, C.N. Pope, JHEP 11, 176 (2015). https://doi.org/10.1007/JHEP11(2015)176 arXiv:1509.07142 [hep-th]

32. Z.Y. Fan, Phys. Rev. D 97(6), 066013 (2018). https://doi.org/10. 1103/PhysRevD.97.066013 arXiv:1801.07870 [hep-th]

33. H. Motohashi, K. Noui, T. Suyama, M. Yamaguchi, D. Langlois, JCAP 07, 033 (2016). https://doi.org/10.1088/1475-7516/2016/ 07/033 arXiv:1603.09355 [hep-th]

34. J. Ben Achour, M. Crisostomi, K. Koyama, D. Langlois, K. Noui, G. Tasinato, Degenerate higher order scalar-tensor theories beyond Horndeski up to cubic order. JHEP 12, 100 (2016). arXiv:1608.08135 [hep-th]

35. E. Babichev, C. Charmousis, A. Cisterna, M. Hassaine, Regular black holes via the Kerr-Schild construction in DHOST theories. JCAP 06, 049 (2020). https://doi.org/10.1088/1475-7516/2020/ 06/049 arXiv:2004.00597 [hep-th]

36. O. Baake, C. Charmousis, M. Hassaine, M.S. Juan, JCAP 06, 021 (2021). https://doi.org/10.1088/1475-7516/2021/06/021 arXiv:2104.08221 [hep-th]

37. O. Baake, M. Hassaine, Eur. Phys. J. C 81, 642 (2021). https://doi. org/10.1140/epjc/s10052-021-09449-2 arXiv:2104.13834 [hepth]

38. O. Baake, M.F.B. Gaete, M. Hassaine, Spinning black holes for generalized scalar tensor theories in three dimensions. Phys. Rev. D 102(2), 024088 (2020). arXiv:2005.10869 [hep-th]

39. T. Kobayashi, N. Tanahashi, Exact black hole solutions in shift symmetric scalar-tensor theories. PTEP 2014, $073 E 02$ (2014). arXiv: 1403.4364 [gr-qc]

40. E. Babichev, C. Charmousis, A. Lehébel, JCAP 04, 027 (2017). https://doi.org/10.1088/1475-7516/2017/04/027 arXiv: 1702.01938 [gr-qc]

41. J. Chagoya, G. Tasinato, JCAP 08, 006 (2018). https://doi.org/10. 1088/1475-7516/2018/08/006 arXiv:1803.07476 [gr-qc]

42. C. Charmousis, M. Crisostomi, R. Gregory, N. Stergioulas, Phys. Rev. D 100(8), 084020 (2019). https://doi.org/10.1103/PhysRevD. 100.084020 arXiv:1903.05519 [hep-th] 
43. E. Babichev, C. Charmousis, Dressing a black hole with a timedependent Galileon. JHEP 08, 106 (2014). arXiv:1312.3204 [gr$\mathrm{qc}]$

44. A. Anabalon, A. Cisterna, J. Oliva, Asymptotically locally AdS and flat black holes in Horndeski theory. Phys. Rev. D 89, 084050 (2014). arXiv:1312.3597 [gr-qc]

45. M. Bravo-Gaete, M. Hassaine, Thermodynamics of a BTZ black hole solution with an Horndeski source. Phys. Rev. D 90(2), 024008 (2014). arXiv:1405.4935 [hep-th]

46. W. Kim, S. Kulkarni, S.H. Yi, Quasilocal conserved charges in a covariant theory of gravity. Phys. Rev. Lett. 111(8), 081101 (2013). arXiv:1306.2138 [hep-th]

47. Y. Gim, W. Kim, S.H. Yi, The first law of thermodynamics in Lifshitz black holes revisited. JHEP 1407, 002 (2014). arXiv:1403.4704 [hep-th]

48. L.F. Abbott, S. Deser, Stability of gravity with a cosmological constant. Nucl. Phys. B 195, 76 (1982)

49. S. Deser, B. Tekin, Gravitational energy in quadratic curvature gravities. Phys. Rev. Lett. 89, 101101 (2002). arXiv:hep-th/0205318

50. S. Deser, B. Tekin, Energy in generic higher curvature gravity theories. Phys. Rev. D 67, 084009 (2003). arXiv:hep-th/0212292

51. A. Herrera-Aguilar, D.F. Higuita-Borja, J.A. Méndez-Zavaleta, Black hole scalarization through spacetime anisotropic scaling symmetry. arXiv:2012.13412 [hep-th]

52. M. Bravo-Gaete, M.M. Juárez-Aubry, Thermodynamics and Cardy-like formula for nonminimally dressed, charged Lifshitz black holes in new massive gravity. Class. Quantum Gravity 37(7), 075016 (2020). arXiv:2002.10520 [hep-th]
53. E. Ayón-Beato, M. Bravo-Gaete, F. Correa, M. Hassaine, M.M. Juárez-Aubry, Microscopic entropy of higher-dimensional nonminimally dressed Lifshitz black holes. Phys. Rev. D 100(4), 044024 (2019). https://doi.org/10.1103/PhysRevD.100.044024 arXiv:1904.09391 [hep-th]

54. M.B. Gaete, L. Guajardo, M. Hassaine, JHEP 04, 092 (2017). https://doi.org/10.1007/JHEP04(2017)092 arXiv:1702.02416 [hep-th]

55. M. Bravo-Gaete, M.M. Juarez-Aubry, G. Velazquez-Rodriguez, arXiv:2112.01483 [hep-th]

56. R.M. Wald, Black hole entropy is the Noether charge. Phys. Rev. D 48(8), R3427 (1993). arXiv:gr-qc/9307038

57. V. Iyer, R.M. Wald, Some properties of Noether charge and a proposal for dynamical black hole entropy. Phys. Rev. D 50, 846 (1994). arXiv:gr-qc/9403028

58. L. Smarr, Phys. Rev. Lett. 30, 71-73 (1973). https://doi.org/10. 1103/PhysRevLett.30.71 (Erratum: Phys. Rev. Lett. 30 (1973), 521-521)

59. F.J.G. Abad, M. Kulaxizi, A. Parnachev, On complexity of holographic flavors. JHEP 01, 127 (2018). arXiv:1705.08424 [hep-th]

60. M. Bravo-Gaete, M.M. Stetsko, Planar black holes configurations and shear viscosity in arbitrary dimensions with shift and reflection symmetric scalar-tensor theories. arXiv:2111.10925 [hep-th]

61. L. Lehner, R.C. Myers, E. Poisson, R.D. Sorkin, Gravitational action with null boundaries. Phys. Rev. D 94(8), 084046 (2016). arXiv: 1609.00207 [hep-th] 